(e-migrinter

e-Migrinter

$10 \mid 2013$

Genre et imbrication des rapports de domination dans les médias des minorités ethniques

\title{
Féminisation des supports médiatiques
}

discours et représentations des femmes immigrées latino-américaines dans les journaux ethniques du Pays Basque

\section{Ana Mendieta}

\section{(2) OpenEdition}

\section{Journals}

Édition électronique

URL : https://journals.openedition.org/e-migrinter/473

DOI : 10.4000/e-migrinter.473

ISSN : 1961-9685

\section{Éditeur}

UMR 7301 - Migrinter

Édition imprimée

Date de publication : 11 avril 2013

Pagination : 49-60

ISSN : 1961-9685

Référence électronique

Ana Mendieta, "Féminisation des supports médiatiques », e-Migrinter [En ligne], 10 | 2013, mis en ligne le, consulté le 20 mai 2021. URL : http://journals.openedition.org/e-migrinter/473 ; DOI : https:// doi.org/10.4000/e-migrinter.473 
Féminisation des supports médiatiques : discours et représentations des femmes immigrées latinoaméricaines dans les journaux ethniques du Pays Basque

Ana Mendieta

Cet article a été traduit de l'anglais par Laura Bortoluzæi-Collingwood.

C et article entend montrer que les journaux destinés aux femmes immigrées latino-américaines résidant au Pays Basque représentent leurs lectrices comme des « protagonistes » actives de leurs parcours migratoires, et non pas comme des victimes de la discrimination au travail ou de la violence domestique, contrairement aux représentations des femmes immigrées dans les médias généralistes/dominants espagnols. La première partie de l'article présente le contexte démographique de la population étrangère recensée en Espagne et dans la Communauté autonome du Pays Basque. Dans la deuxième partie, nous nous intéressons à la création des supports médiatiques qui lui sont destinés en Espagne, avant, dans la partie suivante, de nous centrer sur le cas particulier des supports médiatiques ethniques destinés aux femmes immigrées résidant au Pays Basque. La quatrième partie analyse les représentations dominantes des femmes immigrées dans les médias généralistes en Espagne, très différentes de celles construites dans les journaux ethniques destinés aux femmes immigrées du Pays Basque. Celles-ci seront analysées dans la cinquième et dernière partie de l'article.
Croissance démographique de la population étrangère en Espagne et dans la Communauté autonome du Pays Basque

La création de nouveaux médias diasporiques par et pour les immigrés nouvellement arrivés se développe en Espagne dans un contexte de croissance démographique et d'installation d'une population étrangère. Depuis le 1er janvier 2012, un total de 5,7 millions d'étrangers a été enregistré lors du recensement national espagnol ou «padrón de habitantes». Ces 5,7 millions d'étrangers représentent 12,1\% de la population totale de l'Espagne ${ }^{1}$.

Tableau $\mathbf{n}^{\circ} \mathbf{1}$ : Effectifs d'une sélection de groupes immigrés en Espagne.

\begin{tabular}{|c|c|c|}
\hline & $\mathbf{2 0 0 1}$ & $\mathbf{2 0 1 2}$ \\
\hline Marocains & 233415 & 783137 \\
\hline Équatoriens & 139022 & 306380 \\
\hline Colombiens & 87209 & 244670 \\
\hline Boliviens & 6619 & 184706 \\
\hline \multicolumn{3}{|c|}{ Source : INE 2012. }
\end{tabular}

L'Espagne a rapidement évolué d'un pays qui, historiquement, envoyait des émigrés à l'étranger, vers un pays qui reçoit un nombre croissant d'immigrés. En effet, de 1996 à 2010, le nombre d'immigrés en Espagne a été multiplié par près de dix, passant de 540000 à plus de 5,7 millions (Blanco, 2007, pp. 2-8). Entre 2007 et 2009, le nombre d'étrangers recensés a augmenté de $23 \%$.

${ }^{1}$ Ce sont les chiffres les plus récents (avril 2012) fournis par l'Institut national de la statistique (INE). 
En ce qui concerne les pays les plus représentatifs de l'immigration en Espagne entre 2001 et 2012, les immigrés du Maroc sont passés de 233415 à 783 137, les immigrés de l'Équateur de 139022 à 306380 et les immigrés de Colombie de 87209 à 244670 .

Le nombre d'immigrés marocains et équatoriens a été multiplié par plus de deux au cours de ces neuf dernières années, tandis que les immigrés boliviens sont passés de 6619 à 184 706. Aujourd'hui, le nombre le plus élevé d'étrangers recensés en Espagne provient de Roumanie, avec 895970 personnes ( $f f$. Tableau $\left.n^{\circ} 1\right)$.

La démographie de l'immigration dans la Communauté autonome du Pays Basque $(\mathrm{CAB}$, qui inclut les provinces de Bizkaia, Araba et Gipuzkoa) reflète aussi la croissance que connaît l'Etat espagnol. Les derniers chiffres montrent qu'un total de 151 162 immigrés étrangers est recensé dans la CAB (Ikuspegi, 2012), et qu'ils représentent 6,9\% de la population basque totale (2 191 722 millions). Entre 2005 et 2009, le volume total de la population étrangère recensée dans la Communauté autonome du Pays Basque a augmenté de 81 \% (Ikuspegi, 2009, pp. 1-4). Les principaux pays de provenance sont la Roumanie, le Maroc, la Colombie, la Bolivie, le Portugal, l'Équateur ( $c f$. Tableau $\mathrm{n}^{\circ} 2$ ), et dans une moindre proportion le Brésil, l'Algérie, le Paraguay et la Chine.

Tableau $\mathrm{n}^{\circ} 2$ : population immigrée dans la Communauté autonome du Pays Basque.

\begin{tabular}{|c|c|}
\hline & $\mathbf{2 0 1 2}$ \\
\hline Marocains & 17722 \\
\hline Roumains & 17556 \\
\hline Boliviens & 11657 \\
\hline Colombiens & 11112 \\
\hline Portugais & 8426 \\
\hline Équatoriens & 5648 \\
\hline
\end{tabular}

Source : Ikuspegi, 2012.
Dans ce contexte de croissance de l'immigration étrangère, l'augmentation des supports médiatiques créés pour et/ou par les immigrés eux-mêmes s'inscrit dans une tendance générale de pratiques médiatiques communautaires, alternatives et participatives avec lesquelles les publics sont devenus des producteurs actifs de contenus. Selon Mark Deuze, il n'existe pas seulement une relation naturelle entre l'augmentation de l'immigration et celle des médias ethniques, mais cette croissance doit être intégrée à une émergence mondiale de médias dits communautaires, citoyens, et d'un journalisme qui s'appuie sur les communautés et est connu sous le nom de "grassroots journalism» (journalisme de la base) (Deuze, 2006, p. 263).

Les publics de ces médias ne sont pas seulement des consommateurs, mais aussi des producteurs de contenus dans leur propre contexte culturel, et ils le font en échangeant des commentaires sur des pages web ou en publiant leur propre organe de presse. En effet, les lecteurs se sont octroyés un rôle fondamental dans la production des médias en devenant les principaux sujets d'un journalisme citoyen qui peut atteindre un public mondial (Gillmor, 2006, p. 15). Ainsi, l'expansion d'une culture des médias mondiale et participative dans laquelle il existe une relation plus directe entre les professionnels des médias et leur public explique le succès et l'impact des médias destinés aux immigrés (Deuze, 2006, p. 264).

L'expansion de ces médias pour immigrés reflète aussi l'insatisfaction grandissante des minorités ethniques par rapport à la façon dont elles sont traitées par les médias dominants. Le fait d'associer immigrés d'une part, et crimes et conflits d'autre part, par exemple, est devenu partie intégrante de l'image collective des immigrés véhiculée par la presse espagnole depuis le milieu des années 1990. Plusieurs auteurs ont montré comment certains médias élaborent un discours raciste caractérisé par une stratégie générale de représentation de 
soi positive et une image négative de «l'autre», qui met l'emphase sur les différences, les dérives et les menaces (Nash, 2005, pp. 76-78 ; Lario Bastida in Van Dijk, 2006, pp. 23-29).

Pour leur part, les médias destinés aux immigrés proposent des reportages à la fois sur leur pays d'origine et leur pays d'adoption, et encouragent à la fois la préservation de l'identité des immigrés et leur intégration dans la société qui les accueille. Ainsi, les immigrés deviennent des producteurs actifs de contenus sur leurs propres supports médiatiques (Deuze, 2006, pp. 269-276).

\section{Évolution des supports médiatiques destinés aux immigrés étrangers en Espagne}

Plusieurs études sur les types de médias destinés aux immigrés en Espagne ont été publiées au cours des dernières années dans ce qui reste encore un domaine d'intérêt académique nouveau. Selon Jessica Retis, depuis la fin 2007, la ville de Madrid dispose de 29 journaux et de 35 magazines destinés spécialement aux immigrés (Retis, 2008, pp. 71-120). En Espagne, le nombre de "médias diasporiques", "médias ethniques » ou «médias des migrants» se monte à 300 supports médiatiques, selon Alicia Ferrández Ferrer (Ferrández Ferrer, 2009, pp. 260-261). Dans le $3^{\circ}$ Anuario de la Comunicación del Inmigrante en España 08/09 qui publie les résultats du recensement effectué par l'équipe de recherche MinORITYMEDIA (Migrinter, Université de Poitiers), Laura Navarro comptabilise 330 supports médiatiques destinés aux immigrés en Espagne, dont 135 journaux et magazines, 108 stations de radio et programmes radiophoniques, 36 stations de télévision et programmes télévisés, et 51 pages web, journaux en ligne et stations de radio ou de télévision en ligne (Navarro, 2008, pp. 110-115). Selon Navarro, $53 \%$ des supports médiatiques pour immigrés sont de nature commerciale, et ils sont presque tous diffusés gratuitement. $66 \%$ de ces médias ciblent un groupe spécifique, parmi lesquels $43 \%$ sont destinés aux «LatinoAméricains », $10 \%$ aux «Européens de l'Est », 5\% aux «Arabes », 3,7\% aux «Asiatiques» et 3,1\% aux «Africains» (Navarro, 2008).

En ce qui concerne les publications papier, le pionnier en la matière en Espagne est Pueblo Nuevo, un magazine créé en 1992 à Madrid. Pueblo Nuevo a été suivi par les magazines El Latinoamericano et Euromundo Latino en 1994, et par le magazine mensuel gratuit Ocio Latino en 1995. Une autre édition pionnière est Attawassul, un magazine trimestriel destiné à la communauté arabe créé en 1999 à Barcelone comme projet de l'association socioculturelle Ibn Batuta. Au milieu des années 1990, le journal Hua Xin Bao fut fondé pour aider la communauté chinoise en Espagne, et en 2004 El Mandarín devint la principale publication destinée à la communauté chinoise vivant en Espagne (Etnia comunicación, 2007, pp. 90, 92 ; Retis, 2008).

Mais les médias papier destinés aux immigrés en Espagne acquirent une réelle visibilité lorsque les hebdomadaires Sí, Se Puede et Latino commencèrent à paraittre à Madrid en 2004 et 2005 respectivement (Rosell, 2008, p. 87). L'hebdomadaire gratuit Latino s'adresse spécifiquement aux immigrés latino-américains en Espagne, et a trois éditions à Madrid, Barcelone et Valence. Latino comptabilise une diffusion hebdomadaire totale de 90878 numéros entre janvier et décembre 2009, selon les dernières données fournies par le PGD (Publicaciones Gratuitas Ejemplares Distribuibles), une section de l'Office national de circulation des journaux (OJD). Par contre, Sí, Se Puede (qui a cessé de paraitre fin 2011) s'adressait à tous les immigrés et a eu une diffusion hebdomadaire de 96947 numéros entre juillet 2009 et juin 2010 dans ses trois éditions de Madrid, Barcelone et Valence, selon le PGD. 
En 2005, Copernal Publishing commença la publication du magazine mensuel Raír, qui fut ensuite segmenté en plusieurs éditions s'adressant à différentes nationalités, comme Raír. Rumana pour les Roumains, Raír. Marruecos pour les Marocains, Raí Africa pour les Africains, Raír Ucrania pour les Ukrainiens; ainsi que Raír. Paraguay, Raír. Dominicana, Raír. Perú, Raír. Bolivia, Raí. Argentina, Raí. Ecuador et Raíz. Colombia pour les différentes nationalités latino-américaines. L'ensemble des éditions de Raír a dominé le marché avec une diffusion mensuelle totale de 352083 numéros entre juillet 2009 et juin 2010, selon le PGD.

La communauté roumaine, quant à elle, dispose des publications Român in Lume (Les Roumains dans le monde), Romanul din Spania (Les Roumains en Espagne) et Noi in Spania (Nous, en Espagne), qui sont tous publiés en langue roumaine. Romanul din Spania a eu une diffusion bimensuelle de 23931 numéros entre janvier et décembre 2010, et Noi în Spania a publié 15011 numéros chaque semaine entre janvier et décembre 2011, selon le PGD.

Plusieurs études, notamment le rapport de 2008 sur les médias des migrants (Estudio de Medios para Inmigrantes ou EMI), montrent que $27 \%$ des immigrés en Espagne lisent des hebdomadaires gratuits qui leur sont spécifiquement destinés, et notamment Latino, Si, Se Puede, Noi in Spania, El Comercio de Ecuador et Nova Duma; près de $25 \%$ d'entre eux lisent les principaux quotidiens gratuits 20 Minutos, Metro et Que?, et $16 \%$ lisent des journaux mensuels gratuits pour les immigrés, en particulier Ocio Latino, Raír. Équateur et Raír. Marruecos (EMI, 2008, pp. 1-16).

Globalement, l'objectif principal des médias des immigrés est de proposer une source d'informations sur les questions d'immigration, un espace de représentation sociale pour les immigrés et une plate-forme publicitaire et politique qui mette l'accent sur l'intégration des immigrés et la réussite du processus d'immigration (Retis, 2007, pp. 7,8).

Par ailleurs, pour étudier les représentations des femmes immigrées latino-américaines dans les supports médiatiques qui leur sont destinés, il est également important de connaître les représentations médiatiques des femmes immigrées dans les médias généralistes espagnols.

\section{La couverture médiatique des femmes immigrées dans les médias dominants en Espagne}

Une analyse de presse portant sur plus de vingt des principaux journaux espagnols conduite par le Centre de recherche Mugak de Donostia-San Sebastián entre mars 2006 et novembre 2007 montre que les femmes immigrées ne sont spécifiquement mentionnées que dans $13 \%$ des 30000 articles étudiés.

Par ailleurs, seuls 174 des 1010 articles qui abordent le statut professionnel des femmes $(3,41 \%$ sur un total de 29648 reportages étudiés) se rapportent en particulier au statut professionnel des femmes immigrées. Parmi ces 174 articles, $43 \%$ concernent les femmes immigrées travaillant comme employées de maison et $22 \%$ concernent les femmes immigrées prostituées (Bañón, 2008, pp. 7-20).

Selon Mary Nash, la couverture médiatique des femmes immigrées dans la presse espagnole peut être décrite selon deux concepts principaux: l'invisibilité et le stéréotype. L'invisibilité des femmes immigrées dans les informations présente un paradoxe, étant donné le grand nombre de femmes immigrées qui sont arrivées en Espagne au milieu des années 1990, à une époque où la grande majorité de l'immigration vers l'Espagne provenait d'Amérique latine, et où un tiers des immigrés légaux étaient des femmes. La 
couverture médiatique, cependant, ne reflétait pas cette réalité et les femmes étaient en permanence exclues des articles traitant des immigrés (Nash, 2005, p. 105). L'inexistence des femmes immigrées dans le discours des médias espagnols véhicule une image de celles-ci comme étant un collectif social sans identité ni projection sociale dans la société hôte, ce qui accentue la contradiction entre leur manque de représentation et leur poids démographique important.

D'un autre côté, le discours des médias sur les femmes immigrées a été défini par un autre concept central : la vie de famille, avec la prédominance de l'archétype traditionnel d'une femme mariée, dépendante, passive et marginalisée dans la société. Ainsi, la présence de femmes immigrées dans les informations a été évoquée sous l'angle de la famille, de la maternité et de la reproduction sexuelle, avec peu ou pas de reconnaissance de leur individualité ou du rôle des femmes comme seules figures dominantes de leur propre parcours d'immigration. Étant donné qu'il a surtout été fait référence aux femmes immigrées en tant que femmes enceintes, mères ou épouses, il leur a été attribué le crédit d'avoir contribué à résoudre un problème permanent de faible natalité.

Un autre élément construit l'image des femmes immigrées comme étant «l'autre» dans le discours dominant des médias espagnols: leur conversion en symbole d'une culture différente, qui en fait les supports des valeurs essentielles de leur communauté toute entière et les empêche de créer leur propre parcours d'immigration (Nash, 2005, p. 118). Ainsi, le poids de la culture traditionnelle des femmes immigrées comme signe de violation des droits de la personne est devenu évident avec des reportages qui, depuis le milieu des années 1990, ont condamné les mutilations génitales féminines pratiquées en Espagne par des femmes immigrées de certaines cultures africaines (Nash, 2005, pp. 121-123).
En fait, le prototype des femmes immigrées a souvent été assimilé dans les informations aux femmes musulmanes, ce qui est en contradiction totale avec les données démographiques actuelles de l'Espagne. Depuis janvier 2012, les 2746809 femmes étrangères recensées en Espagne représentent 48,1\% du nombre total d'étrangers dans le pays (5 711040 ). Sur ces 2746809 femmes étrangères, $42 \%$ sont originaires de l'Union européenne, $26 \%$ d'Amérique du Sud et $15 \%$ d'Afrique (INE, 2012).

En ce qui concerne leur statut professionnel, les femmes immigrées ont, depuis le milieu des années 1990, souvent été présentes dans les médias uniquement comme employées de maison, prostituées, ou comme victimes d'exploitation sexuelle, de violence domestique ou de discrimination raciale. Ainsi, le cas de Lucrecia Pérez, d'ascendance dominicaine, assassinée par quatre hommes masqués au cours d'un crime à motivation raciste, qui a fait les gros titres des médias espagnols en 1996 (Nash, 2005, pp. 142-143).

Une conclusion similaire peut être tirée suite à une analyse plus récente, réalisée en 2004 par Asunción Bernárdez Rodal, du portrait des femmes immigrées dans des reportages des principales chaines de télévision espagnoles (TVE-1, La 2, Antena 3 et Telecinco). Les stratégies de représentation des femmes immigrées sont définies par la victimisation, l'exacerbation de leur féminité à travers leur rôle de mères, d'employées de maison, de ménagères et d'épouses, le manque d'individualité (les femmes immigrées n'ont pas de nom, d'appartenance ou de profession), et l'émergence de «la différence» pour insister sur la distance entre « nous » et « elles » (Bernárdez Rodal et al., 2007, pp. 138-140). 


\section{Les supports médiatiques ethniques destinés aux femmes immigrées dans la Communauté autonome du Pays Basque}

La création et l'évolution des médias ethniques pour les immigrés dans la Communauté autonome du Pays Basque et leur ordre du jour médiatique peuvent s'inscrire dans l'interaction entre les demandes culturelles des immigrés et celles des « nations minoritaires » récemment étudiées par Ricard Zapata-Barrero. En Espagne, les minorités nationales ou les « nations minoritaires »- comme préfère les désigner Ricard Zapata-Barrero (2007, p. 4) ne partagent pas forcément la culture politique de la société et de l'Etat dominants. De ce fait, les effets qui sont à l'origine de l'arrivée et de l'installation durable des immigrés dans les "nations minoritaires", comme le Pays Basque ou la Catalogne, sont différents. De ce point de vue, les transformations basées sur l'immigration affectent également les "nations minoritaires ». L'étude de trois auteurs majeurs sur ce sujet - Joseph Carens, Will Kymlicka et Rainer Bauböck - est fondée sur sept postulats de base, selon ZapataBarrero. Tout d'abord, l'intégration des immigrés implique une double transformation dans l'identité des immigrés et dans l'identité des sociétés hôtes. Ensuite, cette intégration ne peut enfreindre les valeurs libérales et démocratiques. Enfin, l'intégration des immigrés doit être faite dans le contexte d'une culture commune publique, et non privée. Le quatrième postulat indique qu'en principe, les attentes des immigrés et des «nations minoritaires » sont contradictoires dans la mesure où les immigrés tendront à s'intégrer dans la culture prédominante, ce qui a pour effet de créer un autre élément de pression sur les « nations minoritaires " au cours de l'émergence de leur nation. Le cinquième postulat assume que, malgré une relation directe entre les politiques d'immigration et la compréhension de la société hôte, cette relation revêt une importance vitale pour les communautés culturelles minoritaires. En fait, Zapata-Barrero avance que, lorsque nous évoquons la gestion de l'immigration pour les " nations minoritaires ", nous abordons l'un des fondements qui légitimisent les demandes d'autonomie gouvernementale des " nations minoritaires ». Le sixième postulat indique que les demandes faites par les immigrés et celles qui sont faites par les administrations autonomes sont compatibles et ne sont pas mutuellement exclusives, mais ont une conception inclusive et convergente. La dernière hypothèse insiste sur le fait que les immigrés qui s'installent dans les "nations minoritaires » sont désavantagés, car les politiques d'immigration des Etats ne fournissent pas aux «nations minoritaires » les outils et ressources leur permettant de gérer l'intégration d'immigrés, qui à son tour peut impacter le développement culturel des " nations minoritaires » (Zapata-Barrero, 2007, p. 7).

Dans ce contexte, Zapata-Barrero souligne que Carens défend le besoin et le droit des «nations minoritaires » à négocier avec les gouvernements fédéraux leur compétence à élaborer des politiques d'immigration qui ne risquent pas d'handicaper la préservation de leurs propres identités en tant que "nations minoritaires». Kymlicka indique aussi qu'une " nation minoritaire » cherchant à préserver une culture sociétale différente doit avoir une maitrise des politiques d'immigration, et que si la « nation minoritaire » ne dispose pas de suffisamment de compétences dans ce domaine, la tendance des immigrés qui s'établissent dans la « nation minoritaire » est de s'intégrer à la culture dominante, ce qui offre en général plus d'opportunités d'emploi, selon Zapata-Barrero.

D'ailleurs, de nombreux supports médiatiques ethniques publiés dans la Communauté autonome du Pays Basque accordent une large place au concept d'intégration dans la société basque. Cependant, le contenu de ces supports 
médiatiques reflète un concept d'intégration qui ne suppose pas l'assimilation complète des femmes immigrées dans la société basque. Ces supports médiatiques dépeignent l'intégration comme un processus lent et progressif d'incorporation des femmes immigrées dans la société basque grâce à leur connaissance croissante de la culture, des villes et du peuple basques. En d'autres termes, les reportages parus dans ces publications couvrent des évènements dans lesquels les femmes immigrées tiennent un rôle prépondérant, dans le but d'élargir leur connaissance de la société basque et ainsi de faciliter leur processus d'intégration dans cette même société. C'est le cas du magazine trimestriel Mujeres del MundoMunduko Emakumeak. (Femmes du monde, en espagnol et en basque). Dans son numéro de novembre 2007, l'article «Viaje a Gernika» (Voyage d'étude à Guernica) raconte l'expérience édifiante d'un groupe de femmes immigrées, originaires pour l'essentiel d'Amérique latine, qui a effectué un voyage d'étude à Guernica, bombardée par l'aviation allemande en 1937. Dans le même ordre d'idées, l'article «Excursión Vitoria» (Voyage d'étude à Vitoria) ${ }^{2}$, publié dans le magazine trimestriel MISSI (Mujeres Inmigrantes de San Ignacio - Femmes immigrées de San Ignacio), montre que la visite et la découverte d'une ville basque peuvent favoriser une meilleure intégration dans la société basque. En même temps, ces publications incluent également des reportages où des femmes immigrées réaffirment leur identité culturelle et participent aux activités permettant d'aider leurs compatriotes restés dans le pays d'origine.

Ici, le concept d'intégration serait plus proche de l'analyse faite par la sociologue de l'Université du Pays Basque Cristina Blanco, qui affirme que les modèles traditionnels d'intégration des immigrés dans les sociétés hôtes, assimilation, "melting-pot» et pluralisme culturel doivent être remis en

${ }^{2}$ Extrait de MISSI - Mujeres Inmigrantes de San Ignacio, diciembre 2007, p. 9. cause, reformulés et élargis parce que : «le monde devient fluide, flexible, et qu'il s'étend le long de, près de et entre les frontières d'Etats-nations, qui sont de moins en moins capables de mettre de l'ordre et d'organiser la vie entre eux » (Blanco, 2008, p. 324). Selon Blanco, l'augmentation des activités transnationales des immigrés entre leur patrie d'origine et leur pays hôte (des transactions financières régulières, par exemple des transferts d'argent, une participation à la vie politique dans leur pays d'origine, etc.), qui leur permettent de construire et de reconstruire leur vie simultanément dans plus d'un endroit, et la formation d'enclaves ethniques comme schéma d'installation volontaire des immigrés dans la société hôte, sont les deux facteurs principaux qui contribuent à la reformulation des paradigmes traditionnels de l'intégration (Blanco, 2008, pp. 325-330).

Le concept d'intégration souffre donc actuellement d'une transformation et d'une évolution vers celui d'identité transnationale par laquelle les immigrés vivent dans leurs sociétés hôtes sans assimilation totale et sans rompre les liens avec leur patrie d'origine. Dans ce contexte, les supports médiatiques ethniques peuvent contribuer à la pérennisation des identités d'immigrés liées au lieu d'origine (Blanco, 2008, pp. 345-346). Ce nouveau concept se reflète dans le contenu des supports médiatiques ethniques qui s'adressent aux femmes immigrées au Pays Basque et qui sont analysés dans le présent article.

\section{La couverture médiatique des femmes immigrées latino- américaines dans les magazines ethniques de la Communauté autonome du Pays Basque}

\author{
Mujeres del Mundo «Babel»-Munduko
} Emakumeak (Femmes du monde), Mujeres con Voz-Emakumeen Ahotsa (La voix des femmes), MISSI (Mujeres inmigrantes de San Ignacio - Femmes immigrées de San Ignacio) 
et Euskadi News sont les principaux magazines qui ont été publiés régulièrement au cours des six dernières années et qui s'adressent spécifiquement aux femmes immigrées, essentiellement originaires d'Amérique latine et d'Afrique, vivant au Pays Basque. Ces quatre magazines ont été sélectionnés pour cette analyse parce qu'ils sont effectivement produits dans des associations créées au Pays Basque et fondées par des femmes autochtones et immigrées. Tous, sauf un, portent un titre commençant par le mot "Mujeres» (femmes). En 2012, MISSI et Euskadi News ont cependant cessé de paraitre.

Une analyse des derniers numéros de ces quatre magazines, tous gratuits, révèle une image des femmes immigrées qui essaie de s'éloigner des stéréotypes et des sujets traités par les médias dominants. Les femmes immigrées dépeintes dans ces magazines ne sont pas des victimes: elles sont les figures dominantes des articles qui leur sont consacrés, ou qu'elles rédigent. Contrairement aux portraits présentés dans les médias dominants, dans ces magazines, les femmes immigrées ont des noms, leur propre identité, et leur propre reconnaissance. En d'autres termes, elles luttent contre l'invisibilité et les stéréotypes grâce à des histoires racontées de leur propre voix. Ce sont des employées de maison, mais elles luttent pour leurs droits. Elles sont parfois victimes de violences, mais elles le racontent avec leurs propres mots et elles exigent que le gouvernement agisse en conséquence.

Ce portrait est conforme aux récentes recherches portant sur le nouveau rôle des femmes immigrées. Le rôle central est désormais tenu par «l'héroïne sacrifiée » qui se caractérise par la réalisation, à la fois, de transferts monétaires et de transferts sociaux. Selon Schwenken, de nombreux auteurs arguent «qu'il est temps de reconnaitre les femmes immigrées comme des agents parce que, en pourcentage, les femmes envoient plus d'argent de leur salaire à la maison que les hommes, elles l'envoient pendant une durée plus longue, et elles orientent cet argent dans des investissements à long terme comme l'éducation et la santé » (Schwenken, 2008, pp. 770-772).

Cependant, parallèlement à ce rôle, l'image de la femme immigrée exploitée (par la traite des femmes) ou de la «belle victime » définit aussi l'image actuelle des femmes immigrées. Ainsi, déclare Schwenken, "la politique et la mobilisation des médias envers les femmes immigrées hésitent actuellement entre deux obsessions : l'évocation de la situation désespérée des femmes exploitées et l'éloge de la générosité des femmes qui envoient la plus grande partie de leurs revenus à l'étranger ». Les deux types de migration, ajoute Schwenken, font partie de «la même logique capitaliste : l'exclusion du monde du travail », qui porte l'idée sous-jacente selon laquelle «il est plus sûr de rester à la maison » (Schwenken, 2008, pp. 772-774).

Cette recherche procède à une description des titres, des sous-titres, des images et des graphiques présents sur les couvertures des derniers numéros disponibles des magazines analysés. Ladite description tente d'étayer l'hypothèse principale: les femmes immigrées ont une voix dans les magazines ethniques publiés à leur intention au Pays Basque, une voix inconnue dans les médias dominants. De plus, les femmes immigrées ont une représentation plus importante comme figures actives dominantes ou comme sujets de leur propre histoire, et pas seulement comme victimes de la discrimination, comme employées de maison, ou comme mères et épouses.

Mujeres del Mundo - Munduko Emakumeak «Babel» (Femmes du monde, en espagnol et en basque) est publié depuis 1995, avec une diffusion de 2000 numéros et de 35 pages qui couvrent des histoires de réussite, de solidarité, de discrimination et de multiculturalisme. Dans son numéro 49 de 
juin 2009, l'article intitulé "Las mijeres de Juárez, no más feminicidios» (Les femmes de Juarez, assez d'assassinats de femmes) est un cri contre les assassinats de femmes dans la ville frontière de Juárez, au Mexique, depuis 1995.

L'article se poursuit en pages 16 et 17 sous le titre «L'État mexicain au banc des accusés », qui renforce l'image des femmes non seulement comme victimes, mais aussi comme actrices du processus judiciaire qui poursuit ceux qui sont responsables des assassinats non encore résolus. L'article insiste sur le silence des autorités mexicaines à propos des meurtres, et sur le fait que les meurtres de trois femmes seulement ont fait l'objet d'un jugement, un processus qui s'est déroulé au Chili à l'époque pour éviter les menaces et l'intimidation qu'aurait entraîné un procès dans la ville de Juárez.

En résumé, cet article pose la question de la recherche de la justice dans un cas où les femmes ont été réduites au silence. Bien que le nombre d'immigrées mexicaines ne soit pas représentatif de toutes les immigrées de la Communauté autonome du Pays Basque, beaucoup d'entre elles peuvent s'identifier au contenu de cette histoire : la réclamation d'un rôle actif, et non d'une victimisation.

Dans d'autres articles de ce numéro de Mujeres del Mundo, les femmes font entendre leur propre voix - dans un article portant sur une conférence sur le féminisme et le milieu universitaire, deux articles sur des groupes de femmes immigrées qui se sont formés dans deux villes basques, et deux pages supplémentaires remplies de poèmes, de recettes et de lettres envoyées au rédacteur en chef par les lectrices. De plus, les trois dernières pages de ce numéro couvrent des informations de service public, en particulier sur des ressources liées à la violence domestique, en espagnol, en anglais et en français.
Mujeres con Voz - Emakumeen Ahotsa (La voix des femmes) est un magazine bilingue en espagnol et en basque qui est publié sporadiquement par l'ONG Círculo Solidario Euskadi, basée à Bilbao. Son numéro de décembre 2007 inclut huit articles, portant sur différents sujets sous le titre «Pour une participation politique et sociale efficace des femmes ». Les sujets couverts par les articles vont du mouvement des femmes zapatistes au Chiapas, Mexique, jusqu'à la création de groupes pour le renforcement de l'autonomie des femmes au Nicaragua, au Salvador et au Sahara. Comme le nom du magazine l'indique, son objectif principal est de donner la parole aux femmes en général et aux femmes immigrées en particulier, ce qui explique le contenu des articles, qui met l'accent sur l'utilité des mouvements de femmes comme outil essentiel d'accès à leur autonomie, et par conséquent, à la parole.

L'article sur la création de centres d'autonomie des femmes dans les provinces basques de Bizkaia et de Gipuzkoa souligne l'importance de ces centres comme outils de participation active des femmes aux activités politiques et sociales, et leur renforcement en tant qu'actrices de la lutte pour l'égalité plutôt qu'en tant que sujets passifs. L'article intitulé «La participation politique des femmes au Nicaragua est très active » traite des accomplissements du groupe de femmes $V$ enancia pour encourager la participation des femmes à la vie politique du Nicaragua. Une autre histoire intitulée «Construire des municipalités plus égalitaires et démocratiques » a pour personnage central une femme salvadorienne, photographiée à Bilbao, qui travaille au Salvador à promouvoir des actions sur l'égalité des sexes dans différentes municipalités du pays.

MISSI Mujeres Inmigrantes de San Ignacio - Femmes immigrées de San Ignacio) était un magazine biannuel publié depuis 1995 par une association de femmes immigrées, essentiellement latinoaméricaines, qui se réunissaient chaque 
semaine dans les bureaux du district municipal de San Ignacio à Bilbao. De petit format, ce magazine, dont le contenu était conçu et écrit par les femmes de MISSI, s'intéressait aux problèmes locaux les concernant, comme les évènements multiculturels, les expositions faites par les femmes, les ateliers de poésie. Un article, tiré du numéro de juillet 2007 de MISSI, couvrait un évènement multiculturel de cuisson de riz baptisé "Arroces del mundo »" (Riz du monde), dans lequel les femmes, une fois encore, tenaient un rôle prépondérant.

Autre magazine destiné aux femmes immigrées du Pays Basque, EuskadiNews-El Magazine de las Culturas (Le magazine des cultures), était quant à lui financé, entre autres, par le gouvernement du Pays Basque. Son premier numéro, paru en janvier 2008, suivait un schéma régulier en termes de sections et de types de sujets. La couverture comportait presque toujours deux photos se rapportant aux interviews principales du numéro, en général avec une femme autochtone et une femme immigrée qui avaient toutes deux réussi. Dans le numéro de décembre 2009, les trois personnes en couverture étaient trois stylistes : deux femmes basques et une femme marocaine. Ces trois femmes représentent une nouvelle génération de stylistes de mode, la mode étant présentée dans l'article comme un facteur d'intégration culturelle. Avec des articles comme celui-ci et d'autres articles sur la culture basque, les voyages autour du monde, les problèmes des femmes, les conseils juridiques, la santé et la beauté, Euskadi News essayait de se positionner comme un magazine de premier plan ayant une influence sur les institutions politiques basques.

\section{Conclusion}

Malgré la crise économique et financière actuelle, les supports médiatiques qui s'adressent aux femmes immigrées de la

${ }^{3}$ Extrait de MISSI - Mujeres Inmigrantes de San Ignacio, julio 2007, pp. 4-5.
Communauté autonome du Pays Basque sont engagés dans un processus de croissance et de consolidation de l'offre.

Un examen du contenu des derniers numéros des magazines Mujeres del Mundo Munduko Emakumeak "Babel», Mujeres con Voz - Emakumeen Abotsa, MISSI et Euskadi News montre que les femmes immigrées ont une représentation plus importante comme protagonistes actives ou sujets des articles qui les concernent, et non comme victimes de discrimination, employées de maison, mères ou épouses. Ainsi, en mettant en lumière une représentation plus diverse/variée des femmes immigrées, ces publications abordent des sujets différents de ceux couverts par les médias dominants. Par exemple, ces magazines destinées aux femmes immigrées de la Communauté autonome du Pays Basque proposent des articles sur les femmes immigrées qui sont des activistes politiques et sociales majeures, des intellectuelles ou des «cols blancs» comme les stylistes de mode.

Cependant, cette tendance à la féminisation n'est pas autant mise en évidence dans le partage du pouvoir de ces médias. Il n'est même pas clairement établi si ces magazines ont des salles de rédaction, des journalistes free-lance ou des rédacteurs pour préparer les contenus, ni combien de femmes y collaborent, étant donné que beaucoup d'articles ne sont pas signés.

Mais cette situation reflète une tendance générale à l'absence de femmes journalistes issues de minorités ethniques dans les médias espagnols, contrairement à ce qui se passe dans d'autres pays européens, selon Lario Bastida. En fait, les femmes espagnoles n'ont pas vraiment eu accès aux salles de rédaction avant les années 1970, lors de l'avènement de la démocratie après la mort de Franco. La présence des femmes journalistes espagnoles dans les salles de rédaction est passée de $28 \%$ en 1995 à $37 \%$ en 2005, mais leur présence à des postes de 
direction dans les médias est toujours de $20 \%$.

Une exception à la règle d'absence complète de femmes appartenant à des minorités ethniques dans les médias espagnols - une absence qui, selon Lario Bastida (2008, pp. 159-180), n'a pas encore fait l'objet d'une étude complète - est le cas de Francine Gálvez. Originaire de Guinée équatoriale, elle est apparue dans des reportages de la chaîne de télévision publique espagnole TVE-1 dans les années 1990 , et a récemment animé une émission culturelle sur La 2, la deuxième chaîne de télévision publique espagnole. Quelques émissions de télévision récentes, comme Televisión sin fronteras (Telemadrid) ou la disparue Con todos los acentos (La 2), ont également été présentées par des femmes immigrées.

Ana Mendieta

MCF en journalisme Faculté des sciences sociales et de la communication Université du Pays Basque anamaria.mendieta@ehu.es
Bibliographie

Arnold, Anne-Katrin; Schneider Beate (2007) Communicating separation? Ethnic media and ethnic journalists as institutions of integration in Germany, Journalism, $\mathrm{n}^{\circ} 8(2)$, pp. 115-136.

Aierbe, Peio (2008) Representación de las mujeres trabajadoras inmigrantes en los medios de comunicación, in Bañón, A. (ed.) Comunicación, empleo y mujer inmigrante, Donostia, Tercera Prensa-Hirugarren Prentsa S.L., pp. 7-20.

Bernárdez Rodal, Asuncion (2007) Mujeres inmigrantes en España. Representaciones en la información y percepción social, Madrid, Fragua, pp. 134-140.

Blanco, Cristina (2008) Procesos migratorios contemporáneos y su incidencia en los tradicionales paradigmas de integración, in García Roca, J.; Lacomba, J. (eds.) La inmigración en la sociedad española. Una radiografía multidisciplinar, Barcelona, Edicions Bellaterra, pp. 323-350.

Blanco, Cristina (2007) Inmigración y política migratoria en España. Líneas maestras y su evolución en el marco de la Unión Europea, in Panfichi, A. (ed.) Aula Magna. Migraciones internacionales, Lima, Fondo Editorial de la Pontificia Universidad Católica del Perú, pp. 2-8.

Deuze, Mark (2006) Ethnic media, community media and participatory culture, Journalism, n³, pp. 263-276.

Etnia Comunicación (2006) $1^{\circ}$ Anuario de la Comunicación del Inmigrante en España, Madrid, Etnia Comunicación, 240 p.

Etnia Comunicación (2007) $2^{\circ}$ Anuario de la Comunicación del Inmigrante en España, Madrid, Etnia Comunicación, 246 p.

Ferrández Ferrer, Alicia (2009) Un nuevo objeto de estudio: los medios de comunicación diaspóricos en España, in Actas, VI Congreso sobre las migraciones en España, A Coruña, Universidade a Coruña, pp. 260-261. 
Georgiou, Myria (2006) Diaspora, Identity and the Media. Diasporic transnationalism and mediated spatialities, Cresskill, Hampton Press, pp. 29-79.

Gillmor, Dan (2006) We the Media. Grassroots journalism by the people, for the people, Sebastopol, O’Reilly Media, p. 15.

Guedes Bailey, Olga (2007) Transnational Identities and the Media, in Guedes Bailey, O.; Georgiou, M. ; Harindranath, R. (eds.) Transnational Lives and the Media, Hampshire, Palgrave MacMillan, pp. 212-230.

Gómez-Escalonilla, Gloria (2008) Voces de la inmigración. Medios latinos en Madrid, Madrid, Universitas, pp. 61-89.

Ikuspegi (2012) General CAPV, por nacionalidades y sexo. Año 2012, Bilbao, Ikuspegi@k.

Instituto Nacional de Estadística (2012) Avance de la Explotación estadística del Padrón a 1 de enero de 2012. Datos provisionales, Madrid, INE [en ligne] URL: $<$ http: $/ /$ www.ine.es $/$ jaxi $/$ menu.do?type $=$ pc axis\&path $=/ \mathrm{t} 20 / \mathrm{e} 245 / \mathrm{p} 04 /$ provi\&file $=$ pca $\underline{\text { xis }}>$.

Lario Bastida, Manuel (2008) Las mujeres periodistas de otras etnias y nacionalidades, in Bañón, A. (ed.) Comunicación, empleo y mujer inmigrante, Donostia, Tercera PrensaHirugarren Prentsa S.L., pp. 159-180.

Lario Bastida, Manuel (2006) Medios de comunicación e inmigración, Murcia, Convivir sin racismo, pp. 23-29.

Mendieta, Ana (2008) Las publicaciones periódicas impresas dirigidas a los inmigrantes latinoamericanos en España. Análisis de estrategias de integración e identitarias, Leioa, Universidad del País Vasco (Trabajo de suficiencia de investigación).

Nash, Mary (2005) Inmigrantes en nuestro espejo. Inmigración y discurso periodístico en la prensa española, Barcelona, Icaria Editorial, pp. 104144.
Navarro, Laura (2008) Los medios de comunicación nacidos de las nuevas migraciones en España, in $3^{\circ}$ Anuario de la Comunicación del Inmigrante en España, Madrid, Etnia Comunicación, pp. 118-123.

Park, Robert (1922) The Immigrant Press and Its Control, New York, Harper \& Brothers, $496 \mathrm{p}$.

Pérez, J. (2007) Viaje a Gernika, Mujeres del Mundo-Munduko Emakumeak, n³8, p. 5.

Retis, Jéssica (2008) Espacios Mediáticos de la Inmigración en Madrid: Génesis y Evolución, Madrid, Observatorio de las Migraciones y de la Convivencia Intercultural de la Ciudad de Madrid, pp. 57-89.

Retis, Jéssica (2007) Mass media and migrations in Spain. Emergence and consolidation of the new media of Latin America diaspora, in $50^{\text {th }}$. International Association for Media and Communication Research, Actas, pp. 7-8.

Rossell, Maria del Mar (2008) La prensa para inmigrantes se profesionaliza, Cuadernos de Periodistas, n¹3, pp. 81-90.

Schwenken, Helen (2008) Beautiful Victims and Sacrificing Heroines: Exploring the Role of Gender Knowledge in Migration Policies, Signs: Journal of Women in Culture and Society, vol. 33, nº 4, pp. 770-776.

Subervi-Vélez, Federico (1986) The Mass Media and Ethnic Assimilation and Pluralism: A Review and Research Proposal with Special Focus on Hispanics, Communication Research, vol. 13(1), pp. 71-96.

Viswanath, Kasisomoyajula ; Arora, Pamela (2000) Ethnic Media in the United States: An Essay on Their Role in Integration, Assimilation and Social Control, Mass Communication and Society, vol. 3, $\mathrm{n}^{\circ} 1$, pp. 4750.

Zapata-Barrero, Ricard (2007) Setting a Research Agenda on the Interaction Between Cultural Demands of Immigrés and Minority Nations, Joumal of Immigrant \& Refugee Studies, vol. 5, nº 4, pp. 1-25. 